\title{
Sindeleros
}

\section{Talento o capacidad excepcional y Talento o capacidad natural Excepcionalidad o naturalidad, inclusión o exclusión ${ }^{1}$}

\author{
Exceptional and natural talent or ability \\ Talent or natural ability from the notion of inclusion and exclusion
}

\begin{abstract}
Autora:
Mónica Andrea Monsalve Ríos ${ }^{2}$

Recibido: 20/06/2019

Aprobado: 02/12/2019
\end{abstract}

1. Artículo de revisión

2. Licenciada en Educación Especial de la Universidad de Antioquia. Maestra de apoyo en la Unidad de Atencion Integral (UAI) Sabaneta. Grupo de Investigación Cognición, Creatividad y Aprendizaje. monyrios@gmail.com

\section{Resumen}

El presente artículo nos acerca a las nociones de diversidad, educación inclusiva y talento o capacidad natural, comocategorías preliminaresyelementos fundamentales en las trasformaciones educativas actuales, y se desprende de la revisión bibliográfica realizada en la investigación "La metacognición como vía para identificar y promocionar el talento o la capacidad en el aula escolar", este artículo pretende no solo identificar bibliográficamente aspectos relevantes de las nociones antes mencionadas, sino además retomar categorías emergentes como la noción de equidad, y talento o capacidad natural, mostrando las concepciones actuales de estos términos, y posibilitando responder algunos interrogantes ¿la educación inclusiva, incluye o excluye? ¿Puede reconocerse el talento o la capacidad desde la diversidad y equidad en el aula escolar?

Palabras clave: capacidad, talento, diversidad. 


\begin{abstract}
This article brings us closer to the notions of diversity, inclusive education, and talent or capacity, as preliminary categories and fundamental elements in current educational transformations. It is derived from the literature review carried out in the research "Metacognition as a way to identify and promote talent or capacity in the classroom ".

This article aims not only to identify bibliographically relevant aspects of the
\end{abstract}

notions mentioned above, but also to take up emerging categories such as the notion of equity, and talent or natural ability, showing the current conceptions of these terms, and making possible to answer some questions: Does inclusive education include or exclude? And, can talent or capacity be recognized from the diversity and equity in the school classroom?

Keywords: Ability, talent, diversity.

\section{Introducción}

Cuando se habla de educar en el siglo XXI, se habla de una sociedad de la innovación, de aulas como espacios de trasformación social, educativa y cultural, de aceptación de la diversidad y de educación con calidad y equidad como propósito para el nuevo milenio. Dicho propósito de educar en la diversidad, con calidad y equidad se convierte en un reto, debido a que generar procesos diversos y de educación inclusiva, implica la concepción de propuestas pedagógicas, espacios adaptados y, sobre todo ajustes actitudinales, metodológicos y curriculares.

Bajo esta perspectiva, la educación inclusiva y el reconocimiento de la diversidad son necesidades latentes del sistema educativo colombiano, dado que existe el convencimiento político de ofrecer una educación con igualdad de oportunidades para todos los estudiantes, teniendo en cuenta sus intereses, posibilidades, expectativas, pero sobre todo sus talentos o capacidades, que de no ser desarrolladas pueden perderse.

\section{Método}

Para el presenta artículo de revisión bibliográfica, se utilizaron buscadores, repositorios, revistas digitales y bases de datos, como: Dialnet, Redalyc, Google Académico, y revistas electrónicas de psicología y educación; además de libros en formato impreso. Las palabras clave que permitieron la búsqueda de información fueron: "talento", "capacidad", "identificación de talentos", "talentos naturales", "educación inclusiva", "diversidad", "integración educativa", e "inclusión educativa ". La información recopilada se sistematizó mediante una matriz bibliográfica, de la cual se extrajeron 50 registros ubicados entre 2002 y 2018, de los cuales se establecieron categorías preliminares como "Talento o capacidad", 
e "Inclusión educativa y diversidad"; del posterior análisis emergen categorías como "Educación para todos", "Equidad”, y “Talentos o capacidades naturales". El artículo se divide en dos hallazgos encontrados: de la excepcionalidad a la naturalidad y, de lo incluyente a lo excluyente, además de un tercer apartado donde se establece una discusión sobre ambos hallazgos.

\section{De la excepcionalidad a la naturalidad}

El talento o la capacidad en Colombia ha sufrido a través de la historia cambios, mediados por la participación teórica e investigativa de otros países; estos cambios se hacen evidentes durante el rastreo de artículos de países como Cuba, España, Chile, Brasil, Perú, Argentina, Estados Unidos, Holanda, y Colombia, en los que por los cambios teóricos, investigativos y culturales, han empezado a orientar la definición del talento o capacidad, las estrategias de atención, los modelos utilizados y los proyectos o programas, hacia la valoración de los intereses y habilidades de los estudiantes. Por lo cual han empezado a encontrar en el aula escolar un espacio primordial para fomentar y fortalecer los talentos o capacidades, sean estas excepcionales o no.

Dentro del rastreo entre los años 2004-2016, se observa una transición de una visión de excepcionalidad hacia la identificación y atención del talento desde los dones, las habilidades y los intereses, siendo estos, susceptibles de ser potencializados hasta que en un momento dado puedan considerarse excepcionales.

En Colombia las orientaciones dadas por el Ministerio de Educación Nacional (MEN) en (2015) permiten direccionar a las diferentes instituciones sobre la atención del talento o la capacidad desde la excepcionalidad, además, presenta la definición, las cinco categorías de talento, define la doble excepcionalidad, y plantea estrategias para su atención; sin embargo, estas orientaciones no terminan de permear el aula escolar desde los intereses, y las habilidades de la diversidad de estudiantes que allí se encuentran. En países suramericanos como Brasil los autores encontrados manifiestan que el trasegar ha estado marcado en sus inicios por la no claridad de los apoyos, ni de los programas necesarios para atender a la población superdotada. Sin embargo, en la actualidad autores como Souza y Soriano (2004), y Mettrau (2010) plantean en sus artículos, la importancia de la educación basada en las habilidades, los intereses y los estilos de aprendizaje; además, resaltan la participación de los maestros en el desarrollo del talento o la capacidad, la creatividad y las habilidades que poseen sus estudiantes, logrando aprendizajes más innovadores y renovadores, buscando así, no solo la excepcionalidad sino también conocer las habilidades, intereses y curiosidades de los alumnos, que posteriormente podrán convertirse en excepcionales o no. 
Desde otro continente Hoogeveen (2008) presenta como la atención en Holanda ha logrado apoyar a los estudiantes talentosos, gracias a investigaciones realizadas por Monks y Heller (1990), con su modelo multidimensional de superdotación. Lo anterior evidencia que en diferentes países la evolución del concepto de superdotación, altas capacidades, talento o capacidad excepcional, ha estado mediada por la investigación y los aportes teóricos realizados, pero desde una mirada de excepcionalidad. Esto puede comprobarse en lo planteado por Velásquez, Escobar y Escobar (2015) quienes en su investigación Estado del arte en el campo de capacidad y talentos excepcionales en Iberoamérica (2004-2014), ponen de manifiesto que no hay una definición clara del talento o la capacidad, o como nombrarlo, ya que estas definiciones se plantean según el momento histórico y los avances teóricos que se desarrollen en el país y, en segundo lugar, que en su mayoría los artículos hacen énfasis en la identificación, mediante la utilización de pruebas de coeficiente intelectual (CI).

Según lo anterior, se reporta una gran preocupación para el fortalecimiento de los talentos o capacidades en el aula escolar, ya que si se toma la visión de excepcionalidad, desde la medición de CI, la diversidad de la población que se tiene en el aula no tendrá derecho a que se potencien sus habilidades e intereses, pues el acceso a dichas pruebas formales no es amplio. Andreucci (2012) cuestiona la noción de enseñanza descontextualizada de las condiciones, predisposiciones y campo crítico del aula y plantea la importancia de repensar la noción de talento o capacidad únicamente desde la excepcionalidad, al igual que desde la visión de identificación mediante pruebas formales.

Teniendo en cuenta lo expuesto anteriormente, la identificación y caracterización de talentos o capacidades desde la excepcionalidad, mediante pruebas formales, ocupa muchos de los artículos encontrados, Gómez, Avendaño y Lozano (2013) pretenden diseñar y efectuar el análisis psicométrico de una prueba para evaluar talento. Cerchiaro, Paba, y Sánchez (2005) plantean la identificación, caracterización y atención de jóvenes con talentos o capacidades excepcionales, por medio de la aplicación de pruebas formales. Y González y Domínguez (2015) proponen una serie de indicadores para la identificación basados en modelos teóricos de autores como Renzulli con su modelo de los tres anillos, Gagné desde el modelo diferenciado de superdotación, Sternberg con el modelo cognitivo y Ericsson con su modelo de practica deliberada.

Lo ya expresado, nos lleva a pensar que si bien las pruebas diagnósticas se convierten en un insumo válido para la identificación del talento o la capacidad, es necesario articularlas a procesos de identificación no formales y aplicables al ámbito escolar, que permitan mayor reconocimiento de las habilidades, 
potencialidades, e intereses de cada estudiante. Carreras, Arroyo, Valera, (2006) crean un protocolo en el cual se plantea el enriquecimiento de los talentos dentro del ambiente escolar y no por fuera, mediante la modificación de contenidos del currículo, del contexto de aprendizaje, cambios metodológicos, evaluación de los procesos intelectuales, cambios en la estructura y temporalidad de las clases, motivación, y creatividad en el aula; es decir, articulando la identificación del talento o la capacidad en el aula escolar y los elementos que intervienen en ella y, reconociendo allí, los intereses y habilidades de los estudiantes. Por lo cual se considera, que la identificación formal o no formal del talento o la capacidad excepcional o no, deben estar enmarcados en una visión de derecho en la cual se favorezca el potencial, la habilidad, y la capacidad, desde la identificación temprana de capacidades, con el fin de evitar un futuro fracaso escolar (Alonso 2008). En el mismo sentido, Comes, Díaz, Luque, Moliner (2008) y Cabrera y Udaquiola (2013), plantean la identificación como una respuesta eficaz, que permite a los estudiantes, padres de familia y docentes, conocer aspectos familiares, personales, condiciones cognitivas, sociales y emocionales, que se convierten en formas de potencializar los talentos o capacidades que existen en la diversidad del aula; esta identificación estará no solo acompañada de mediciones cuantitativas, sino también de observaciones en el aula, de entrevistas con docentes, padres de familia, pares y con el mismo estudiante, desde una visión cualitativa.

Así mismo, en la revisión bibliográfica se encuentran cuatro proyectos de autores como Hierro (2011), García, Llantada y Mena (2011), Cabrera (2011) y Núñez y Guarín (2012), quienes centran su atención en la identificación educativa, la sistematización y la evaluación de dicha identificación desde una visión de excepcionalidad. Sin embargo, se encuentra en uno de ellos, una visión cambiante de la identificación formal a una basada en componentes ludo-pedagógicos necesarios para estimular, explorar e identificar los diferentes intereses, habilidades e interrogantes. Dichos proyectos ponen de manifiesto como algunos de los autores dejan de lado la excepcionalidad para enfocarse en el desarrollo de habilidades, intereses y gustos.

Sin embargo, en la búsqueda realizada se encuentran artículos cuya postura se contrapone a la identificación del talento o la capacidad mediante test. Lorenzo, Lorenzo y Jiménez (2015), en su artículo titulado: "Y si nos olvidamos de la detección del talento... y si individualizamos el proceso de desarrollo de su talento", ponen de manifiesto cómo el descubrimiento de un talento, se ha considerado como algo mágico, que se visualiza y predice con la aplicación de un test. Los autores plantean que la identificación debería entonces centrarse no solo en el resultado de un test estandarizado, sino tener en cuenta condiciones físicas, biológicas, sociales, de resolución de problemas, de entrenamiento y 
de autogestión; en resumen, pasar de la “excepcionalidad a la naturalidad". Este artículo permite formular preguntas como ¿qué pasa con los talentos o capacidades que no pueden acceder a un test, o prueba? ¿qué pasa con los talentos o capacidades que no se evalúan y que por ende no se desarrollan?

Las respuestas a estos interrogantes, al igual que la noción de talento o capacidad excepcional ha venido modificándose con los cambios culturales y sociales. Se pretende dar respuesta a la diversidad que se acoge en las aulas escolares y que demanda de la educación, la individualidad, la diversidad, la equidad, pero también la naturalidad en los procesos de enseñanza y aprendizaje. Estos interrogantes pueden responderse desde los diferentes modelos actuales, en los cuales se contemplan no solo los talentos o capacidades identificadas por pruebas formales, sino los apoyos necesarios para que el talento o la capacidad natural florezca.

En este sentido, el florecimiento del talento o la capacidad natural, se empieza a encontrar en los artículos desde el 2008 con autores como Tourónm y Campion (2013), Renzulli, (2008), y Gagné, (2015), quienes plantean no solo que la escuela debe responder a las necesidades educativas de los escolares, sino que es necesario que el proceso de enseñanza respete los ritmos de aprendizaje, además del desarrollo del currículo para todos.

Siguiendo la línea de una educación para todos, con equidad y teniendo en cuenta la diversidad de la población, pero centrándose en un modelo aplicable al interior del aula, los autores Renzulli, (2008) con su Modelo de Enriquecimiento Escolar y Gagné (2015) con el Modelo de Desarrollo de Capacidades Naturales (DMNA), se salen del abordaje tradicional de los talentos o capacidades, no desde la excepcionalidad sino desde los intereses, y los talentos innatos, y se convierten en teóricos centrados en el acto de aprendizaje, mediado y representado por las interacciones que tienen lugar entre los aprendices, los profesores y el currículo; dichos modelos proponen que las capacidades naturales pueden llegar a ser excepcionales, si se potencializan. Dichos modelos se complementan con autores como Coyle (2009) quien plantea que cada persona tiene talentos naturales susceptibles de ser desarrollados, por medio de la práctica intensa, el apoyo del docente y la motivación.

Si bien ya se ha abordado la importancia de la identificación y de modelos acordes con la atención del talento natural; de igual manera, se hace importante visualizar. También, las estrategias de atención han variado en virtud de dar respuesta a la diversidad de la población utilizando la resolución de problemas, la expresión artística, el enriquecimiento curricular, el pensamiento crítico y las tecnologías de la información y la comunicación, como estrategias para la atención oportuna 
de los talentos o capacidades naturales. Autores como Leyva, García, Torres (2016) y Rojas, Jiménez y Mora (2009), han utilizado estrategias didácticas que se convierten en una alternativa para la estimulación del talento académico, teniendo en cuenta la motivación, la creatividad y el avance intelectual. En este mismo sentido, Cornelio, Ruiz, Patiño, Vargas, y Calderón (2007) describen la construcción de estrategias pedagógicas que permiten aportar a la atención de la diversidad. Otros autores como Castro, Méndez, Ripoll, y Robles, (2009), Cáceres y conejeros (2008) y Dixon, F., Prater, K., Vine, H., Wark, M., Williams, T., Hanchon, T., y Shobe, C. (2004), plantean el pensamiento crítico y creativo como una fórmula para potenciar e identificar los talentos y capacidades en las aulas, y describen conceptos de educación desde una visión actual que obliga a pensar en formas diferentes de enseñar y aprender, utilizando el pensamiento crítico como herramienta que posibilita la preparación de los estudiantes para el mundo; resaltan además, el papel del cerebro como elemento para la adquisición del aprendizaje, la creatividad y la imaginación.

Otros artículos encontrados retoman las posibilidades del enriquecimiento escolar en el aula de clase como elemento para fomentar el talento y la capacidad. En este sentido, autores como Conejeros, Arenas, Contreras y Gómez (2008) y Chávez, Zacatelco y Acle (2009) ofrecen otra posibilidad, y es la creación de estrategias de enriquecimiento como modelo para fortalecer y desarrollar los talentos y capacidades; se encuentran adicionalmente, proyectos como el realizado por Calvo y Cruz (2005) en el cual plantea la importancia de las inteligencias múltiples para desarrollar la inteligencia lógico-científica.

Otro elemento relevante encontrado en los artículos es el reconocimiento del docente como potenciador de los talentos o capacidades naturales; esto se valida, por medio de autores como Pérez (2012), González y Moreno (2013), Cárdenas (2010), Vilaseca (2006), quienes resaltan la labor docente como fundamental desde dos ámbitos, el pedagógico y el investigativo. La tarea entonces del docente es generar en el ámbito pedagógico prácticas que le permitan a los diferentes talentos o capacidades naturales florecer, además de la exploración de sus habilidades, intereses y gustos con el fin de potenciarlos; es decir, centrar su praxis no en lo mínimo sino en el máximo potencial del estudiante, a partir de sus intereses; así mismo, reconocer en la diversidad y la equidad formas válidas para atender a los estudiantes en el aula de clase, desde sus ritmos y estilos de aprendizaje, y buscar además, metodologías que integren el juego, baile, el arte, la ciencia, la corporalidad y la filosofía. Y, desde el ámbito investigativo, es fundamental un docente que indague, sea curioso y explore, porque esto le permitirá la generación de nuevas miradas y nuevos aprendizajes con relación al talento o la capacidad en el aula escolar. 


\section{De lo incluyente a lo excluyente}

En esta categoría se encontraron artículos de países como España, Costa Rica, México, Chile, Argentina, Estados Unidos y Colombia, entre el 2002- 2018. En el rastreo y revisión de los artículos se encuentran elementos históricos, eventos que ocurrieron internacionalmente para llegar a la construcción de la noción de inclusión, y se plasman elementos discordantes en la concepción, aplicación y visualización de la inclusión como estrategia para incluir o excluir, en los cuales surgen nociones como la diversidad y la equidad, en respuesta a las desigualdades sociales. De igual manera, se encuentran por un lado posiciones gubernamentales y legislativas y, por otro, posiciones académicas, investigativas y teóricas.

Desde la posición legislativa se retoma el artículo de Beltrán, Martínez y Vargas (2015), en el cual presentan un análisis comparativo de las políticas y estrategias desarrolladas en España y adoptadas por Colombia. Esta comparación pone de manifiesto que, si bien Colombia avanza en el reconocimiento de la educación inclusiva, es necesario garantizar las condiciones de permanencia de los alumnos e incentivar medidas eficaces para la atención de otras poblaciones minoritarias, que poco se atiende, según sus intereses y necesidades; además, es necesario sensibilizar a la sociedad para la valoración de las diferencias y la reducción de las brechas sociales.

La reducción en las desigualdades sociales es el punto fundamental que se encuentra en muchos de los artículos revisados; autores como Parra (2011), Araque y Barrio (2010) y Booth y Ainscow (2002) retoman la evolución de la noción de inclusión como elemento de articulación entre prácticas educativas y políticas.

Si bien, la inclusión educativa significó un avance en términos de atención y participación de poblaciones, la diversidad y la equidad llegan en el momento actual con la premisa del respeto y valoración del otro, lo que permite dar respuesta a las desigualdades sociales, educativas y culturales. Sin embargo, para llegar al respeto, validación y comprensión del Otro, la noción de inclusión tuvo un largo trasegar desde aspectos históricos; que comenzaron con una perspectiva en la cual, las personas con discapacidad eran segregadas, escondidas, y no se consideraban sujetos de participación social. Posteriormente, nacen las escuelas especiales con el fin de dar respuesta a la atención desde una visión un tanto terapéutica; luego aparece la obligatoriedad en el ámbito educativo para la atención a estudiantes con dificultades en el aprendizaje, con un principio de normalización, donde los estudiantes debían adquirir comportamientos normales para convivir en la sociedad. El principio de normalización avanza y 
se convierte en el principio de aceptación y, desde esta perspectiva, se ofrecen servicios y se garantizan los derechos educativos desde modelos de integración escolar, en los cuales el estudiante debía adaptarse al ambiente educativo. Desde esta época se nombra a dichos estudiantes desde el termino de Necesidades Educativas Especiales (NEE). Luego, el desarrollo teórico y práctico dio el paso de la educación integradora a la inclusión; así, organismos como la UNESCO, y elementos como la Declaración de Salamanca, la reunión de Jomtien entre otros, fueron decisivos para plantear la inclusión como un tema que permea todos los ámbitos del quehacer educativo.

Sarto y Venegas (2009) reconocen el concepto de educación inclusiva desde un enfoque de derecho, en el cual es necesaria la creación de estrategias de enseñanza-aprendizaje, la colaboración de la familia y el entendimiento de la escuela como espacio de convivencia. Posteriormente, cobra importancia el término de atención a la diversidad con un enfoque inicial de reconocimiento de la diversidad de estudiantes en las aulas escolares; y desde allí, garantizar el derecho a la educación, reconocer las necesidades individuales, combatir la desigualdad y adoptar modelos más flexibles, bajo la premisa del respeto.

Durante el rastreo se encuentran una serie de artículos que plantean cómo la educación inclusiva presenta nuevos retos desde la atención a la diversidad, la convivencia, la justicia social y la educación para “todos", lo que pone de manifiesto las carencias que enfrenta la educación inclusiva actualmente.

La concepción de la educación inclusiva presenta en la actualidad puntos de encuentro y de desacuerdo, algunos de ellos orientados a que es una educación basada en la justicia social, la ética, la democracia y, no, en una visión caritativa, mercantilista y de cobertura. Esta educación acoge a "todos" los sujetos de derecho entre los que se encuentran las diversas poblaciones consideradas minorías (Escudero y Martínez, 2010). Sin embargo, “todos” tienen derecho a la educación obligatoria pero no "todos" tienen derecho a la educación debida, no "todos" pueden acceder con equidad y justicia curricular que es lo que promulga la educación inclusiva, para poder así responder a interrogantes como: ¿Es inclusivo el termino de inclusión?, ¿se habla de "todos" pero también de "algunos"? (Echeita, Duk, Calderon y Skliar, 2018). En este sentido, es válido responder que la educación inclusiva es un proceso, por lo tanto, es necesario crear condiciones de educación con calidad, con la participación activa de todos los implicados en el proceso, y así, establecer principios de equidad, solidaridad e igualdad de oportunidades. (Calvo y Verdugo, 2012). 
Otro elemento relevante en la educación inclusiva, el cual requiere claridad, es en qué contenidos o temas deben ser inclusivos;sobre todo, conociendo el énfasis que la educación ha puesto en el desarrollo de competencias básicas obligatorias, en las cuales se estandariza el aprendizaje; desde allí, la educación inclusiva tiene como objetivo la sensibilización frente a las necesidades, talentos, capacidades e intereses, lo que hace necesario superar la visión desde la competitividad y la eficiencia. De igual manera, sucede con escuela como espacio de inclusión y de exclusión, ya que por un lado, debe responder a los cambios propuestos por las entidades gubernamentales por medio de la expedición de decretos que imponen nuevas trasformaciones, sin dar tiempo de asimilar otras; pero, por otro lado, es la manera de categorizar, estigmatizar y excluir.

Algunos autores encontrados plantean que la educación inclusiva debe definirse desde las voces de la exclusión, esto permitiría hacer cambios a partir de principios éticos, y asumir la justicia como sustento, al ofrecer la posibilidad de centrar la atención no solo en el concepto de inclusión sino de promover una educación de calidad para todos, desde el concepto de participación y personalización (Echeita y Domínguez 2011),

Lo ya descrito, plantea el reto de reconocer y atender a la diversidad de la población en el aula escolar, no solo desde la atención y comprensión, sino desde la diversidad, la equidad y la justicia social. Sin embargo, en algunos artículos se observa con mayor énfasis el tema de la discapacidad, pero poco se habla de la educación inclusiva desde el talento o la capacidad en el aula escolar. Desde una visión de excepcionalidad, esto pone de manifiesto las dificultades para incluir y excluir en el aula de clase a los estudiantes tanto por su dis-capacidad como por su talento o capacidad.

Autores como Giraldo y Núñez (2008), Giraldo (2008) y Giraldo, (2010) presentan una serie de propuestas en las cuales el talento o la capacidad pueden encontrarse dentro del aula escolar, pero además pueden ser visualizados, valorados y comprendidos, si se tienen todas las orientaciones. Uno de los artículos propone la utilización del Plan de Atención al Talento en la Inclusión (PATI), que busca la trasformación de la escuela y la atención con calidad al talento en el aula escolar; estas iniciativas abren el camino para la trasformación de la escuela hacia el reconocimiento y atención del talento. Valadez y Avalos (2008) plantean una serie de interrogantes sobre: ¿cómo puede la escuela orientar al talento? ¿cómo pueden intervenir los padres y docentes? y ¿cómo realizar procesos efectivos de identificación y evaluación?, con el fin de dar respuesta a estos interrogantes se retoma el modelo de habilidades naturales de Gagné $(1985,1997,2009)$ y se resalta 
la importancia de la educación inclusiva desde la convivencia, así mismo, se establece la necesidad de prácticas diferenciadas, la modificación de procesos de enseñanza, de contenidos y del currículo con base en las inteligencias múltiples.

\section{Excepcionalidad o naturalidad, inclusión o exclusión}

Colombia, como muchos otros países, sigue avanzando en el tema de reconocer la diversidad en la escuela, no solo como un proceso educativo, sino de impacto social, cambio que es fundamental para lograr una conciencia social transformadora. Sin embargo, aunque se considera la inclusión como proceso trasformador que reduce la exclusión, en ocasiones es un proceso excluyente, ya que no podemos hablar de inclusión de una población por una o varias características, sin excluir a otras.

Por lo tanto, el respeto y reconocimiento de la diversidad y la equidad como noción, se convierten actualmente en la base para eliminar todo tipo de discriminación y barreras del aprendizaje, es decir, el cambio en el pensamiento será reflejado en el cambio de actitud.

Desde esta perspectiva, cuando asumimos una educación basada en lo cotidiano, en lo diverso, en la comprensión de ese Otro, y le damos importancia al reconocimiento de ese Otro como ser que participa y convive en una sociedad, de un nosotros, se podrán generar de manera más positiva y real prácticas educativas que trabajen por romper los estereotipos, y eviten la discriminación.

El reconocer ese otro, desde su pensamiento, accionar pero sobre todo desde su potencial, académico, cognitivo, creativo, crítico y social, abriría caminos diferentes a los ya planteados desde la base de derechos básicos o mínimos, pero no sobre la base del fortalecimiento de talentos o capacidades, las cuales dan la posibilidad de fomentar aprendizajes, valores y competencias, que le permitan a los estudiantes la participación en diferentes contextos con posiciones críticas, políticas y sociales.

Por lo tanto, es necesario tener en cuenta que las trasformaciones y los cambios educativos, se originan a partir de cambios sociales y culturales, pero también actitudinales. Una verdadera trasformación social radica en el reconocimiento del otro como ser semejante, con el cual se puede, no solo convivir en armonía, sino interactuar y relacionarse; por lo tanto, la educación en la actualidad deberá responder a la necesidad de educar en la diversidad y con equidad de manera crítica; educar en aprender a aprender; enseñar a pensar, y pensar al aprender; 
en donde se estimule el potencial del estudiante y se busquen metodologías y estrategias diferenciadas que permitan el desarrollo adecuado de cada uno, y que velen por el reconocimiento de sus dificultades, pero también de sus capacidades.

Es necesario reflexionar entonces, sobre cómo la equidad cobra mayor relevancia en el ámbito escolar, cuando se reconoce que en la diversidad de la población confluyen tanto dificultades como potencialidades, siendo estas últimas, de las más olvidadas en la escuela, por lo cual es necesario centrar la atención en los intereses, gustos, y pasiones más que en las mediciones, y diagnósticos.

Dicha reflexión debe realizarse desde lo humano, entendiendo que el camino para identificar y potenciar el talento o la capacidad, debe venir no solo de la propia reflexión y entendimiento de cada individuo sobre su potencial, sino además de cómo se valora este potencial en el ámbito escolar, para generar procesos adecuados de atención a la diversidad.

En este sentido, la idea de la superdotación como un rasgo simple, innato, no cambiante, ha sido eclipsada por una noción multidimensional y sujeta a desarrollo y cambio. El foco de atención se ha desplazado, según Treffinger y Feldhusen (1996), a las aptitudes específicas y las capacidades que surgen en áreas particulares de talento. Es mucho más valioso y útil reconocer los puntos fuertes de un talento específico (manifiesto o emergente), que simplemente afirmar que un niño es superdotado. Solo a través de la educación, en la familia y en la escuela, junto con un sustancial esfuerzo del niño, el talento emerge, se desarrolla y crece. La escuela debe centrarse en la identificación y desarrollo del talento en todos los niños, y adecuarse a los diferentes niveles y dominios de talento para proporcionarles los servicios que respondan a sus necesidades (Tourón, 2004 p. 9).

En síntesis, el talento o la capacidad podrán florecer, si se enriquecen con experiencias escolares y familiares. Por lo cual, la escuela deberá ser un lugar abierto, flexible y dinámico donde no se incluya, sino que se aprenda a convivir con el potencial de la diversidad de la población. 


\section{Conclusiones}

- Asumir la diversidad desde la normalidad, desde lo natural, nos permitirá pensar en políticas y en acciones prácticas que repercutirán en los cambios de paradigmas basados en principios de equidad, diversidad, donde convergen nociones de convivencia y justicia social.

- Colombia ha hecho esfuerzos significativos por establecer decretos, y reglamentaciones para garantizar el derecho a la educación para "todos"; esto ha implicado avances y retos, ya que supone comprender la escuela como espacio de construcción y reconocer las individualidades, necesidades, dificultades, talentos o capacidades. El reto no se reduce a adaptar la escuela desde lo físico, para dar cabida a un determinado grupo de alumnos, sino que demanda un proceso de reestructuración y co-construcción global de la escuela desde lo curricular, metodológico, y lo convivencial, para responder a la diversidad (Parrilla, 2002).

- Las investigaciones revisadas muestran la importancia de reconocer el fortalecimiento del talento o la capacidad en el aula de clase como una prioridad en temas de atención a la diversidad; así mismo, se vislumbra al maestro como actor fundamental en el fortalecimiento de los talentos o capacidades y, en general, resaltan la importancia de generar propuestas novedosas con el uso de tecnología, pero también con la implementación de estrategias que le permitan al estudiante conocer su propia aprendizaje y hacerlo más autónomo.

- Se requieren docentes centrados en las necesidades de los estudiantes, donde prime el contacto tanto social como emocional, donde se planteen objetivos de aprendizaje claros que le permitan fortalecer al estudiante sus talentos o capacidades; esto implica un docente que reconozca particularidades del estudiante en las dimensiones cognitiva, afectiva y social, en las diferentes edades o niveles educativos, y que elabore estrategias que permitan descubrir y descubrirse. 


\section{Referencias}

Andreucci, P. (2012). El talento: una construcción en y desde la pedagógica dialógica. Psicoperspectivas individuo y sociedad. Recuperado de http://www. psicoperspectivas.cl/index.php/psicoperspectivas/article/view/200/240

Alonso, J. (2008). La educación de los alumnos con sobredotación intelectual, VII Congreso Bienal de la FICOMUNDYT, 37-64.

Araque, N y Barrio, J. (2010). Atención a la diversidad y desarrollo de procesos educativos inclusivos. Ciencias sociales, (4), 1-37.

Beltrán, Y., Martínez, Y. y Vargas, A. (2015). El sistema educativo colombiano en el camino hacia la inclusión. Avances y retos. Educ.Educ. 18(1), 62-75.

Booth, T, y Ainscow, M. (2002). Guía para la Evaluación y mejora de la educación inclusiva. Consorcio Universitario Para la Educación Inclusiva. Universidad Autónoma de Madrid, Retomado de http://www.ite.educacion. es/formacion/materiales/126/cd/unidad_3/material_M1/guia_indicadores_ inclusiva_unesco.pdf

Cabrera, P. (2011). ¿Qué debe saber y saber hacer un profesor de estudiantes con talento académico? Una propuesta de estándares de formación inicial en educación de talentos. Estudios pedagógicos. Recuperado de https://scielo. conicyt.cl/scielo.php?script=sci_arttext\&pid=S0718-07052011000200002

Cabrera, E, y Udaquiola, C. (2013). Recuperado de http://www.scielo.org.co/ pdf/rups/v15n2/v15n2a11.pdf

Cáceres, P, y Conejeros, M. (2008). Efecto de un modelo de metodología centrada en el aprendizaje sobre el pensamiento crítico, el pensamiento creativo y la capacidad de resolución de problemas en estudiantes con talento académico. Revista española de pedagogía, 248, 39-56.

Calvo, C., \& Cruz, I. (2005). El Proyecto Spectrum: aplicación y actividadesde aprendizaje de ciencias en el primer ciclo de la Educación Primaria. Obtenido de https://dialnet.unirioja.es/servlet/articulo?codigo=2057193

Calvo, M, y Verdugo, M. (2012). Educación Inclusiva ¿Una realidad o un ideal? EDETANIA. (41), 17-30. Universidad de Salamanca.

Cárdenas, C. (2010). Concepciones de los maestros del Instituto Marco Fidel Suárez de la ciudad de Manizales, frente a las capacidades excepcionales. Plumilla educativa, 7 (1), 283-296. 
Carreras, L., Arroyo S., y Valera, M. (2006) Protocolo de identificación de niños/as con altas capacidades intelectuales e intervención en estos casos. En: I jornadas nacionales sobre escuela y altas capacidades. Barcelona: Universitat de Barcelona.

Castro, E., Méndez, Z., Ripoll, M., \& Robles, C. (2009). El pensamiento crítico, los talentos excepcionales y las TIC en la Educación. Revista Educación y Humanismo, (17), 142-153. Obtenido de www.unisimonbolivar.edu.co/ publicaciones/index.php/educacionyhumanismo

Cerchiaro, E., Paba, C., \& Sánchez, L. (30 de 1 de 2005). Excepcionalidad y talento: otra comprensión del ser humano para hacernos más humanos. Revista de la facultad de ciencias de salud. Obtenido de https://dialnet. unirioja.es/descarga/articulo/4788260.pdf

Conejeros, M., Arenas, A., Contreras, C. y Gómez, M. (2008). Diseño de un modelo de observación de clases de un programa para alumnos talentosos. Revista Ideación, 28, 152-165

Cornelio, S., Ruiz, R., Patiño, J., Vargas, C., \& Calderón, G. (2007). Una mirada al talento proyecto desarrollo del talento. Bogotá, Colombia: Ediciones Buinaima

Comes, G., Díaz, E., Luque, A., y Mollner, O. (2008). La evaluación psicopedagógica del alumnado con altas capacidades intelectuales. Educación inclusiva, 1, 103-117

Coyle, D. (2009). Las claves del talento. Barcelona, España: Zenith-Planeta.

Chávez, B., Zacatelco, F., \& Acle, G. (2009). Programa de enriquecimiento de la creatividad para alumnas sobresalientes de zonas marginadas. Obtenido de Electronic Journal of Research in Educational Psychology: https:/www. researchgate.net/publication/263765938_Programa_de_enriquecimiento_ de_la_creatividad_para_alumnas_sobresalientes_de_zonas_marginadas

Dixon, F., Prater, K., Vine, H., Wark, M., Williams, T., Hanchon, T., \& Shobe, C. (2004). Teaching to Their Thinking: A Strategy to Meet the CriticalThinking Needs of Gifted Students Revista para la educación de los superdotados, 28, (1), 56-76.

Echeita, G, y Domínguez, A. (2011). Educación inclusiva. Argumentos, caminos y encrucijadas. (17), 23-35. Universidad de Salamanca. Retomado de http:// revistas.usal.es/index.php/0214-3402/article/view/8393

Echeita, G., Duk, C., Calderon, I., Skliar, C. (2018). Conversando sobre educación inclusiva desde dos orillas oceánicas. Dykinson e-book. (IX), 200-220. España. 
Escudero, J, y Martínez, B. (2011). Educación inclusiva y cambio escolar. Iberoamericana de educación, (55), 85-105. Universidad de Murcia. Retomado de

https://rieoei.org/historico/documentos/rie55ao3.pdf

Gagné, F. (2015). De los genes al talento: la perspectiva DMGT/CMTD. Revista de educación. Recuperado de https:/www.mecd.gob.es/ $\mathrm{dctm} /$ revista-de-educacion/articulos368/de-los-genes-al-alento. pdf?documentId=0901e72b81e8ccbo

García, R., Llantada, M., \& Mena, G. (2011). Estrategia para el desarrollo del talento propuesta de ARGOS. Faisca, 5, 17-26. Obtenido de https:/dialnet. unirioja.es/servlet/articulo? codigo $=2477701$

Giraldo, J. (2010). Inclusión y talento en el aula. Inclusión y talento equidad en una educación con calidad.18-23, Universidad Nacional de Colombia

Giraldo, G. (2010). Inclusión y talento en el aula. Inclusión y talento equidad en una educación con calidad.65-74, Universidad de Nova Miami.

Giraldo, J. (2010). Inclusión y talento en el aula dos caras de la una misma moneda. Inclusión y talento equidad en una educación con calidad.75-93, Universidad Nacional de Colombia

Gómez, M., Avendaño, B., \& Lozano, M. (2013). Diseño y análisis psicométrico de una prueba para evaluar talento en estudiantes de trece a dieciséis años. Cultura, Educación y Sociedad, 4(2), 61-88.

González, N., \& Moreno, O. (Enero-Junio de 2013). Una ventana a la enseñanza en niños con capacidades excepcionales en la escuela formal. Revista Quaestiones Disputatae-temas en debate(12), 95-116. Obtenido de http:// revistas.ustatunja.edu.co/index.php/qdisputatae/article/view/268

González, M, y Domínguez, F. (2015). ¿Existen indicadores para identificar el talento? Aula, 21, 21-32. Universidad de salamanca.

Hierro, J. (2011). Orienta2: material para la respuesta educativa del alumnado con necesidades específicas de apoyo educativo por presentar altas capacidades intelectuales. Obtenido de Delegación territorial de educación de huelva eoe especializado en altas capacidades intelectuales.: http://www. juntadeandalucia.es/averroes/centros-tic/21700381a/helvia/aula/archivos/ repositorio/1250/1367/AACC.pdf

Hoogeveen, L. (2008). Los alumnos talentosos en Holanda, VII Congreso Bienal de la FICOMUNDYT. 102-121. Universidad de Salamanca. 
Leyva, J., \& Ortiz, E. (2016). La identificación y estimulación del talento en estudiantes de la universidad de Holguin, Cuba. Pedagogía Universitaria, 21, 1-15.

Lorenzo, A., Lorenzo, J., y Jiménez, S. (2015). Y si nos olvidamos de la detección del talento y si individualizamos el proceso de desarrollo de su talento. Aula, 21,105-127.

MEN. (2015). Documento de orientaciones técnicas, administrativas y pedagógicas para la atención educativa a estudiantes con discapacidad en el marco de la educacion inclusiva. Bogotá-Colombia.

MEN. (2017). Decreto 1421. Bogotá-Colombia. Recuperado de http://es.presidencia.gov.co/normativa/normativa/DECRETO\%201421\%20DEL\%20 29\%20DE\%20AGOSTO\%2ODE\%202017.pdf

MEN. (2018). Derechos Basicos De Aprendizaje. Obtenido de http://aprende. colombiaaprende.edu.co/es/siemprediae/86404

Mettrau, M. (2010). Programas académicos para la formación de profesores de alumnos con superdotación y talento. 13 (1). Retomado de http://www. aufop.com

Núñez, C, y Guarín, D, (2012). Programa de inclusión y talento en el aula. (Tesis de Maestría). Universidad Pedagógica Nacional, Bogotá, Colombia.

Parra, D. (2011). Educación inclusiva: un modelo de diversidad humana. Educación y desarrollo social. 5(1), 139-150. Universidad Militar "Nueva Granada”.

Parrilla, A. (2002). Acerca del origen y sentido de la educación inclusiva. Revista Educación, (327). Retomado de https://sede.educacion.gob.es/ publiventa/detalle.action? $\operatorname{cod}=10520$

Pérez, G. (2012). Descubrir talentos en educación básica primaria: una obligación docente. Uni-pluri/versidad, 12 (3), 75-86. Recuperado de file://D:/Descargas/15159-50551-1-PB.pdf

Renzulli, J. (2008). La educación del sobredotado y el desarrollo del talento para todos. Revista de Psicología, 1, 23-44. Recuperado de https:// vdocuments.mx/reuven-feuerstein-570f9f64a188a.html

Rojas, S., Jiménez, W., y Mora, L. (2009). El uso de la resolución de problemas como instrumento para la caracterización de talento en matemáticas. Asocolme. Recuperado de https://core.ac.uk/download/pdf/12341288.pdf

Sarto, M y Venegas, M. (2009). Aspectos clave de la Educación Inclusiva. Publicaciones del INICO Colección Investigación Salamanca. 
Souza, D y Soriano, E. (2004). La educación de niños con talento en Brasil. La educación de niños con talento en Iberoamérica. Unesco.

Tourónm, J., \& Campión, J. (2013). Atención a la diversidad y desarrollo del talento en el aula. El modelo DT-PI y las tecnologías en la implantación de laflexibilidad curricular y el aprendizaje al propio ritmo. Pedagógica, 256 , 441-459.

Tourónm, J. (2004). “De la Superdotación al Talento: Evolución de un paradigma”. En: Jiménez, C. Pedagogía Diferencial. Diversidad y Equidad. Madrid: Pearson Educación.

Valdez, M, y Avalos, A. (2010). Atención educativa a alumnos sobresalientes, talentosos en escuelas inclusivas. Inclusión y talento en el aula. Inclusión y talento equidad en una educación con calidad.25-35, Universidad Nacional de Colombia

Velásquez, L., Escobar, C., y Escobar, L. (2015). La producción académica en el campo de capacidades y talentos excepcionales en Iberoamérica (2004-2014). Tesis de doctorado Pontificia Universidad Javeriana, Bogotá, Colombia.

Vilaseca, G. (2006). Actitud y práctica educativa del profesorado hacia las necesidades educativas especiales de superdotación. I jornadas nacionales sobre escuela y altas capacidades. Barcelona:Universidad de Barcelona. 